To be published in IEEE ICME, Taipei, June 2004. (C) IEEE.

\title{
Optimal FEC Rate for Media Streaming in Active Networks
}

\author{
Dan Jurca and Pascal Frossard \\ Signal Processing Institute \\ Swiss Federal Institute of Technology Lausanne, Switzerland \\ \{dan.jurca, pascal.frossard $\} @ e p f l . c h$
}

\begin{abstract}
This paper addresses the problem of optimal channel rate allocation for media streaming in active networks, where intermediate nodes are able to perform basic FEC decoding/encoding operations. FEC performance is analyzed in the case of hop-by-hop FEC protection, and compared with an end-to-end FEC scenario, in order to demonstrate the benefits of FEC operations in the intermediate nodes. An optimization problem is formulated, based on a distortion model for video streaming over lossy channels. Finally, the two streaming scenarios are compared in the particular case of MPEG-4 video, under a constrained endto-end delay. FEC operations in intermediate nodes are shown to become especially useful when the links on the streaming path have quite heterogenous characteristics.
\end{abstract}

\section{Introduction}

Due to the heterogeneity of network infrastructures like the Internet, media or video streams often travel heterogeneous links along their path from the streaming server to the clients. For example, the available bandwidth can be very large between two successive nodes, while the quality of service on the next hop can be poor. In this context, the non-trivial task of optimizing the quality of service offered to the end user can obviously benefit from basic operations performed in the intermediate network nodes. Active networks can for example help to improve the quality of service of delay-sensitive media streaming in error-prone environments, by isolating packet losses and trying to recover information already lost within the network, with simple FEC decoding/recoding operations. Then, an optimal video quality can only be guaranteed by a proper allocation of the streaming rate between source information, and error control redundancy.

Rate allocation problems have been studied in various streaming scenarios over passive networks. The joint video source and FEC rate allocation problem has been studied for

This work has been supported by the Swiss National Science Foundation example in [1] in a one-to-one transmission scenario. The authors in [3] propose a novel rate-allocation scheme used with FEC, in order to minimize the probability of packet losses in bursty networks, for a scenario comprising one receiver and multiple senders. Optimal allocation of packetlevel and byte-level FEC is discussed in [2], for the case of video multicast over wired and wireless networks, while the authors of [4] solve the optimal FEC assignment, for scalable video transmission over burst error channels with loss rate uplink. This paper considers the scenario where intermediate nodes are capable of low complexity operations, like FEC encoding/decoding, and presents the benefits of such a method. It extends the analysis of optimal FEC rate allocation for the case of active networks and proposes an optimal source and channel rate allocation strategy for video streaming with total rate and delay constraints.

The paper is organized as follows : Section 2 formulates the optimal rate allocation problem, with the help of a simple distortion model for lossy media streaming. Section 3 analyzes the performance of FEC mechanisms for the cases of transparent networks (end-to-end optimal FEC protection) and active networks (hop-by-hop optimal FEC protection). In Section 4 the two streaming strategies are compared in the particular case of MPEG-4 encoded video streams, and Section 5 concludes the paper.

\section{Problem Formulation}

In general, the distortion perceived at the receiver is caused by the lossy compression of the original information (source distortion) and by packet loss and delays in the network (loss distortion). Under the commonly accepted assumption that distortion caused by losses, and distortion due to quantization are uncorrelated, the end-to-end distortion can be written as :

$$
D=\chi_{R}\left(R_{v}\right)^{\xi}+\chi_{L} R_{v} \pi_{v},
$$

where the first term of the sum represents the source distortion, and the second term is the loss distortion. The source distortion in common encoding schemes (e.g. MPEG) is driven by the encoding rate, and the parameters $\left(\chi_{R}\right.$ and $\left.\xi\right)$ are dependent on the scene content. The loss 


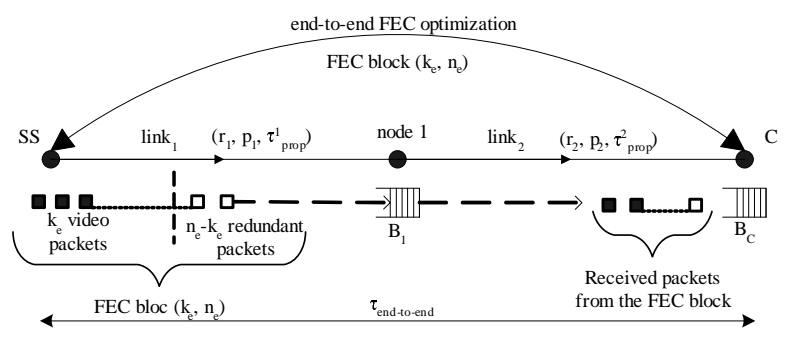

Figure 1. End-to-end FEC scenario

distortion is also driven by the media rate, the loss probability $\pi_{v}$, and a parameter $\chi_{L}$ which depends on the sequence and the encoding format. Allocating a share of the available rate for error correction data represents a natural solution to prevent an uncontrollable increase of the loss distortion. The optimal distribution of the total rate between source information and channel protection is the core of the problem considered here.

Among all error correction techniques, packet-level FEC is generally preferred in the case of multicast-like or delay sensitive transmission scenarios. Generically, a FEC block of $n$ packets contains $k$ media packets and $n-k$ FEC packets. The receiver can fully reconstruct the original $k$ data packets as long as it correctly receives at least $k$ packets of the FEC block. The scenario under consideration becomes the following. A streaming media server (SS) sends live or stored media content to a receiver (C). The media (e.g., video) is encoded and sent through the network in blocks of packets. The video packets are protected with FEC packets, forming FEC blocks. All packets (media and FEC) have an average size of $M$ bytes, and the encoding format allows each data packet to be decoded independently from the others, possibly with some distortion (i.e., we use all received video packets).

We consider a network path between the server and the client made of multiple links $l_{i}$ that connect intermediate nodes $i-1$ and $i$. The intermediate nodes are able to perform FEC encoding/decoding operations. The intermediate nodes $i$ and the client have buffers $B_{i}$, assumed to be large enough to prevent overflow. The server is aware of the main characteristics (available bandwidth $r_{i}$, packet loss probability $p_{i}$ and propagation delay, $\tau_{p r o p}^{i}$, all considered static) of all the links $l_{i}$ along the path to the client. Within this context, two scenarios are studied, where the intermediate nodes either transparently forward packets, or provide simple FEC operations. These scenarios are represented in Figure 1 and Figure 2, respectively.

The end-to-end quality optimization problem can be stated as follows. Given (i) the characteristics $\left(r_{i}, p_{i}\right.$ and $\tau_{\text {prop }}^{i}$ ) of all links $l_{i}$, and (ii) a maximum end-to-end delay $\tau_{\max }$ in the transmission of one video packet, find the optimal transmission scenario $S^{*}$, or equivalently the optimal FEC parameters $\overrightarrow{k^{*}}$ and $\overrightarrow{n^{*}}$, that minimize the end-to-end distortion $D$ :

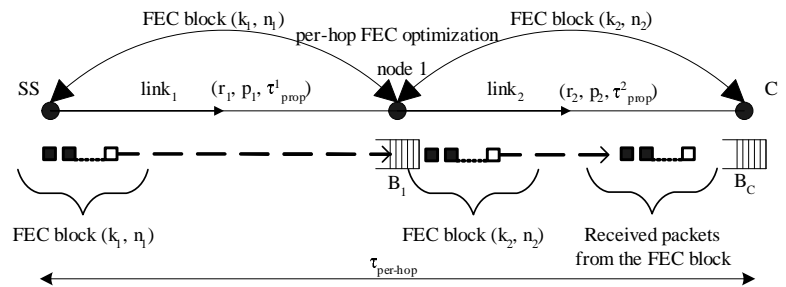

Figure 2. Per-hop FEC scenario

$$
D^{*}=\min _{\vec{k}, \vec{n}}\left(\chi_{R} R_{v}(\vec{k}, \vec{n})^{\xi}+\chi_{L} R_{v}(\vec{k}, \vec{n}) \pi_{v}(\vec{k}, \vec{n})\right),
$$

under the constraint $R_{v} \leq \min \left(r_{i}\right)$ and a maximum transmission delay below $\tau_{\max } .(\vec{k}, \vec{n})$ represent the vectors of FEC parameters for the links in the streaming path.

The next section presents an analytical study of the loss probabilities and transmission delays in the two streaming policies, that will eventually allow to solve the optimization problem. It concentrates on a simple network topology where the path from the server to the client consists of two links and one intermediate node. However, the study can easily be generalized to any topology with multiple hops.

\section{FEC Performances}

\subsection{End-to-End FEC Protection}

In the case of end-to-end FEC protection in a topology like the one in Figure 1, the server sets the parameters $(k, n)$ based on its knowledge about the network status. The intermediate node acts as a simple router and transparently forwards the received packets on the second link. Hence, the media rate is equivalent to: $R_{v}=\frac{k}{n} \min \left(r_{1}, r_{2}\right)$ and the transmission delay becomes:

$$
\tau(k, n)=\tau_{\text {prop }}^{1}+\tau_{\text {prop }}^{2}+\frac{n M}{\min \left(r_{1}, r_{2}\right)},
$$

where $\frac{n M}{\min \left(r_{1}, r_{2}\right)}$ represents the transmission time of a complete $n$-packet FEC block. Without loss of generality, we assume here that the time required for FEC coding can be neglected.

The video loss rate $\pi_{v}$, as seen by the receiver after FEC recovery is expressed as: $\pi_{v}=\frac{\sum_{i=1}^{k} i p_{i}(k, n)}{k}$, where $p_{i}(k, n)$ is the probability of losing $i$ video packets on the two links, after FEC recovery. It is computed as the probability of losing $i$ video packets and at least $\lfloor n-k-i+1\rfloor$ FEC packets, on either the first or the second link. For a uniform and independent loss process, it yields :

$$
\begin{array}{r}
p_{i}(k, n)=\sum_{a=0}^{i}\left(\begin{array}{c}
k \\
a
\end{array}\right)\left(\begin{array}{c}
k-a \\
i-a
\end{array}\right) p_{1}^{a} p_{2}^{i-a}\left(1-p_{1}\right)^{k-a}\left(1-p_{2}\right)^{k-i} \\
\sum_{j=0}^{n-k} \sum_{b=\lfloor c-i+1\rfloor}^{c}\left(\begin{array}{c}
n-k \\
j
\end{array}\right)\left(\begin{array}{l}
c \\
b
\end{array}\right) p_{1}^{j} p_{2}^{b}\left(1-p_{1}\right)^{c}\left(1-p_{2}\right)^{c-b},
\end{array}
$$


where $p_{1}$ and $p_{2}$ respectively represent the loss probability on the first and second link, and $c=n-k-j$.

The extension of the two links case to the more general case of $N$ links and $N-1$ intermediate routers is straightforward. The media rate is equivalent to : $R_{v}=$ $\frac{k}{n} \min \left(r_{1}, \ldots, r_{N}\right)$ and the transmission delay becomes: $\tau(k, n)=\sum_{i=1}^{N} \tau_{\text {prop }}^{i}+\frac{n M}{\min \left(r_{1}, \ldots, r_{N}\right)}$. The expression of $p_{i}(k, n)$ does not have a closed form and we omit it due to lack of space.

\subsection{Hop-by-hop FEC Protection}

In the case of hop-by-hop FEC protection, the losses can be isolated on the various links, at the price of a possible larger end-to-end delay. The server and the intermediate nodes can set different FEC parameters $\left(k_{i}, n_{i}\right)$, individually for each link $l_{i}$ (see Figure 2). The sizes of the FEC blocks are however constrained by a maximum end-to-end delay. The media rate is given by $R_{v}=\min \left(\frac{k_{1}}{n_{1}} r_{1}, \frac{k_{2}}{n_{2}} r_{2}\right)$, and the total delay can be written as :

$$
\tau\left(k_{1}, n_{1}, k_{2}, n_{2}\right)=\tau_{\text {prop }}^{1}+\tau_{\text {prop }}^{2}+\frac{M}{R_{v}}\left(k_{1}+k_{2}\right)+\tau_{w}^{1},
$$

where $\frac{M}{R_{v}}\left(k_{1}+k_{2}\right)$ represents the transmission time of the FEC blocks $\left(k_{1}, n_{1}\right)$ and $\left(k_{2}, n_{2}\right)$ on the first and respectively second link. If the loss probability on the first link is larger than 0 , there is a non-zero probability that the intermediate node waits forever before it receives enough media packets to fill in $k_{2}$ slots in the $n_{2}$-packet FEC block. To avoid such a scenario, a limit is set in the intermediate node, that will send available data after $\tau_{w}^{1}$. We set this limit to be equivalent to the average waiting time in the intermediate node, $\tau_{w}^{1}=\left\lfloor\frac{k_{2}}{k_{1}\left(1-\pi_{v}^{1}\left(k_{1}, n_{1}\right)\right)}\right\rfloor$. Experiments have shown that this value is in general sufficient to absorb the packet losses on the first link. In the very low probability case where the waiting time is larger than $\tau_{w}^{1}$, the FEC parameters on the second link can be slightly different than $\left(k_{2}, n_{2}\right)$, with a small impact on the hop-by-hop FEC performance.

Since the loss processes on the two links are isolated due to the FEC decoding/encoding operations at the intermediate node, the overall media loss rate, as seen by the receiver can be expressed as:

$\pi_{v}\left(k_{1}, n_{1}, k_{2}, n_{2}\right)=\pi_{v}^{1}\left(k_{1}, n_{1}\right)+\pi_{v}^{2}\left(k_{2}, n_{2}\right)\left(1-\pi_{v}^{1}\left(k_{1}, n_{1}\right)\right)$,

where $\pi_{v}^{1}\left(k_{1}, n_{1}\right)$ and $\pi_{v}^{2}\left(k_{2}, n_{2}\right)$ are the video loss rates after FEC recovery on each individual link. They are given by $\pi_{v}^{i}(k, n)=\frac{\sum_{j=1}^{k} j p_{j}(k, n)}{k}$, where $p_{j}(k, n)$ is the probability of losing $j$ media packets out of the FEC block $(k, n)$ after FEC recovery (for each link $i$ ). In the case of a uniform and independent loss process, and with $f=n-k$, $p_{j}(k, n)$ can be written as: $p_{j}(k, n)=\left(\begin{array}{l}k \\ j\end{array}\right) p^{j}(1-p)^{k-j} \sum_{l=\lfloor f+1-j\rfloor}^{f}\left(\begin{array}{l}f \\ l\end{array}\right) p^{l}(1-p)^{f-l}$.

For the general case of $N$ links and $N-1$ intermediate nodes the media rate is: $R_{v}=\min \left(\frac{k_{1}}{n_{1}} r_{1}, \ldots, \frac{k_{N}}{n_{N}} r_{N}\right)$ and the total delay becomes: $\tau(\vec{k}, \vec{n})=\sum_{i=1}^{N}\left(\tau_{\text {prop }}^{i}+\right.$ $\left.\frac{M}{R_{v}} k_{i}\right)+\sum_{i=1}^{N-1}\left\lfloor\frac{k_{i+1}}{k_{i}\left(1-\pi_{v}^{i}\left(k_{i}, n_{i}\right)\right)}\right\rfloor$. In the same time, the overall media loss rate can be expressed as: $\pi_{v}(\vec{k}, \vec{n})=$ $\pi_{v}^{1}\left(k_{1}, n_{1}\right)+\sum_{i=2}^{N} \pi_{v}^{i}\left(k_{i}, n_{i}\right) \prod_{j=1}^{i-1}\left(1-\pi_{v}^{j}\left(k_{j}, n_{j}\right)\right)$.

Armed with expressions for $R_{v}(\vec{k}, \vec{n}), \pi_{v}(\vec{k}, \vec{n})$ and $\tau(\vec{k}, \vec{n})$, we can solve the optimization problem (1). Knowing that FEC performs better with the increase in the block size, we can implement an efficient search algorithm for the optimal solution by limiting the feasible search space for the $(\vec{k}, \vec{n})$ parameters. The search space of the $\vec{n}$ parameters is greatly reduced based on the delay constraint $\tau_{\text {max }}$, while the search space for the $\vec{k}$ parameters is limited knowing the loss probabilities $p_{i}$ on all the links.

\section{Experimental Results}

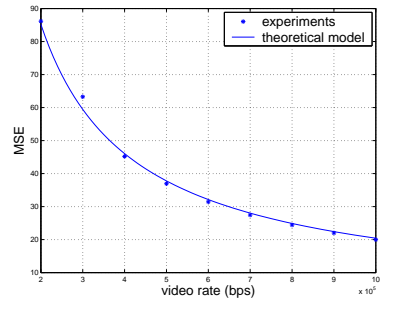

(a) RD-curve, $p=0$

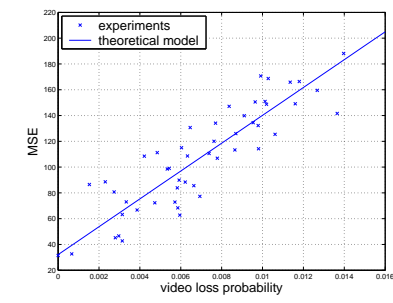

(b) video rate $=600 \mathrm{kbps}$
Figure 3. Validation of the theoretical distortion model for $\chi_{R}=4.3214 \cdot 10^{6}, \xi=-0.8876$ and $\chi_{L}=18 \cdot 10^{-3}$

This section now applies the here-above analysis to the particular case of the MPEG-4 video streaming, where the decoder implements basic error concealment functions. The foreman.cif sequence (300 frames) is encoded at $30 \mathrm{fps}$ with an interval of 15 frames between I-frames, and the packet size is set to $500 \mathrm{~B}$. We first validate the source ratedistortion characteristic and the loss distortion model proposed in Section 2. Figure 3(a) presents the comparison between our theoretical model and the experimental results in the case of no loss, while Figure 3(b) shows the distortion as a function of the packet loss probability for a given video rate. It can be seen that the experimental data fits quite well the analytical values, and similar behavior has been observed for different video rates.

We now solve the optimization problem given from Eq. (1), and find the optimal $(\vec{k}, \vec{n})$ parameters for the hopby-hop FEC protection policy. They are then compared to 


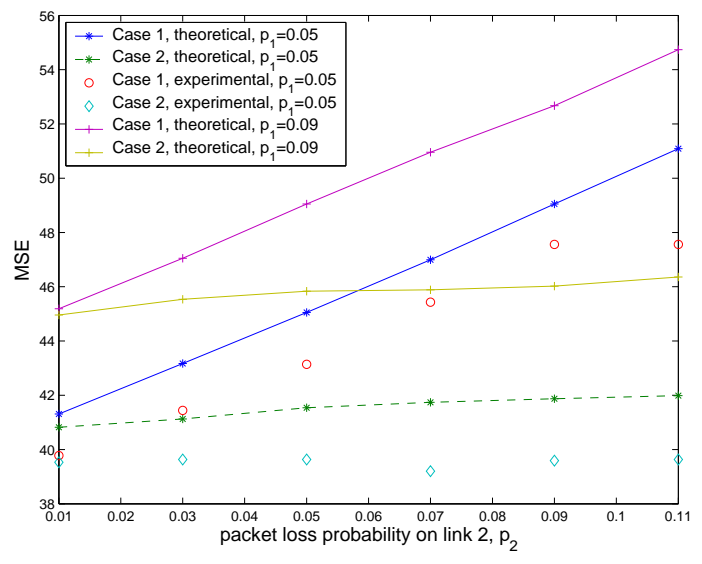

(a) $r_{1}=600 \mathrm{kbps}, r_{2}=1 \mathrm{Mbps}$

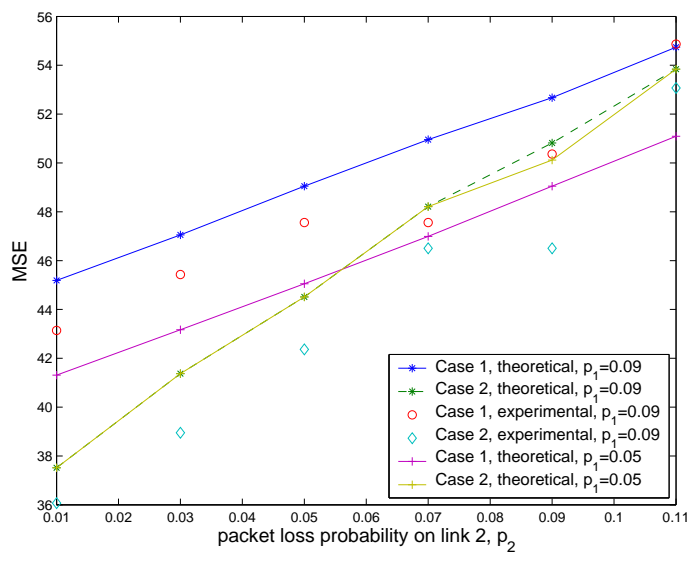

(b) $r_{1}=1 \mathrm{Mbps}, r_{2}=600 \mathrm{kbps}$

Figure 4. Minimal distortion in end-to-end and hop-by-hop FEC scenarios

\begin{tabular}{|c|c|c|c|c|c|c|c|c|c|}
\hline \multicolumn{2}{|c|}{ link 1 } & \multicolumn{2}{|c|}{ link 2 } & \multicolumn{2}{c|}{ Case 1 } & \multicolumn{4}{c|}{ Case 2 } \\
\hline$r_{1}$ & $p_{1}$ & $r_{2}$ & $p_{2}$ & $k$ & $n$ & $k_{1}$ & $n_{1}$ & $k_{2}$ & $n_{2}$ \\
\hline \hline 700 & 6 & 400 & 1 & 15 & 20 & 9 & 17 & 8 & 9 \\
\hline 500 & 2 & 700 & 7 & 18 & 25 & 13 & 16 & 7 & 12 \\
\hline 800 & 5 & 800 & 5 & 29 & 40 & 15 & 21 & 13 & 18 \\
\hline 1000 & 9 & 600 & 3 & 20 & 30 & 12 & 25 & 11 & 14 \\
\hline 600 & 5 & 1000 & 9 & 19 & 30 & 17 & 22 & 6 & 12 \\
\hline
\end{tabular}

Table 1. Optimal $(\vec{k}, \vec{n})$ for end-to-end (Case 1) and hop-by-hop (Case 2) FEC protection, as a function of $r_{i}$ [kbps] and $p_{i}[\%]$.

the optimal parameters for the end-to-end FEC scenario. Table 4 presents the optimal values in the two cases for different parameters of the streaming path segments, where the maximal end-to-end delay has been set to $\tau_{\max }=0.2 \mathrm{~s}$ and the propagation delays have been neglected. It can be seen that the FEC blocks are in general much smaller in the end-to-end case because of the end-to-end delay constraint. Also, the optimal FEC construction greedily uses all the available bandwidth on the highest rate links, in order to limit as much as possible losses on this particular link. Loss therefore occurs almost exclusively on the smaller rate segment.

Figure 4 compares the optimal performance of both FEC techniques in terms of average MSE distortion for different link parameters. As expected, the hop-by-hop protection performs much better than the end-to-end FEC policy. This is especially true for segments with very different characteristics, and the performance becomes similar when the path becomes homogeneous. Also, for stringent end-to-end delay constraints, it can happen that the end-to-end FEC protection performs better thanks to the increased flexibility in building longer blocks. It can be noted finally that the experimental results are slightly better than the theoretical ones. This phenomenon is due to a so low effective loss proba- bility (thanks to the very good FEC protection), that even a high number of simulations can hardly reproduce. In the very small probability case where a FEC block cannot be decoded, the distortion becomes however high enough for the average behavior to jump on the theoretical curve.

\section{Conclusions}

This paper presents a comparison between hop-by-hop and end-to-end optimal FEC protection in active networks. We use the extended capabilities of intermediate nodes to derive a new hop-by-hop FEC coding strategy, and compare it with the classic end-to-end FEC optimization technique. The performance of both strategies are compared in the case of MPEG-4 video streaming on a simple two-segment path topology. Our proposed FEC optimization technique outperforms the classic one when the links in the streaming path have heterogenous parameters, and when the end-toend delay constraint is not too restrictive. In future work, we will develop the proposed FEC technique for a more generic packet loss model, and test the impact of the average burst length onto video quality.

\section{References}

[1] P. Frossard and O. Verscheure. "Joint Source/FEC Rate Selection for Quality-Optimal MPEG-2 Video Delivery". IEEE Transactions on Image Processing, vol.10, no.12, 2001.

[2] T.-W. A. Lee, S.-H. G. Chan, Q. Zhang, W.-W. Zhu, and Y.-Q. Zhang. "Optimal Allocation of Packet-Level and Byte-Level FEC in Video Multicast over Wired and Wireless Networks". In Proceedings of the IEEE Globecom'01, 2001.

[3] T. Nguyen and A. Zakhor. "Distributed Video Streaming with Forward Error Correction". In Proceedings of the Packet Video Workshop, Pittsburg, PA, 2002.

[4] Y.-C. Su, C.-S. Yang, and C.-W. Lee. "Optimal FEC Assignment for Scalable Video Transmission over Burst Error Channel with Loss Rate Uplink". In Proceedings of the Packet Video 03, Nantes, France, 2003. 